7/07/2016

Dr. Marilena V Iorio

Fondazione IRCCS Istituto Nazionale dei Tumori

Start Up Unit, Department of Experimental Oncology and Molecular Medicine

Via Amadeo 42

Milan 20133

Italy

Re: CAN-16-0140R1

MiR-9 and miR-200 regulate PDGFRß-mediated endothelial differentiation of tumor cells in triple negative breast cancer

Dear Dr. lorio:

I am pleased to inform you that your above-referenced manuscript has been accepted for publication in CANCER RESEARCH.

You will receive your proofs electronically within 2 to 3 weeks. If you expect your e-mail address to change within that time, please notify the CANCER RESEARCH Editorial Office (cancerres@aacr.org). The e-mail you will receive will include detailed information about marking and returning your proofs. Please read, correct, and return proofs within 2 business days.

IMPORTANT: Please note that if proofs are not returned within this time frame, publication of the article may be delayed until the next available issue.

Please also note that the assignment to which section of the journal your article will appear is made at the Editors' discretion.

PROCESSING CHARGES: 
New in 2016. In an effort to avoid discouraging authors from providing sufficient detail about their work, the AACR journals transitioned from page charges to a flat publication fee, regardless of page count. If your manuscript was originally submitted on or after January 1, 2016 you will be required to pay a flat publication fee based on the journal and article type and an additional handling fee for each color figure. Manuscripts originally submitted before January 1, 2016 will be charged based on the previous, per-page fee structure.

ONLINE FIRST:

Please note that all AACR journals now offer OnlineFirst publication of accepted manuscripts. The version of the manuscript which will be used for OnlineFirst publication is the PDF version of the manuscript which has been peer reviewed but not yet copyedited or typeset. Please review your accepted manuscript now and reply to this message within 48 hours if you object to having your accepted manuscript posted online in its present form. After proof corrections have been returned, the final edited version of your manuscript, incorporating any corrections or changes made at the proof stage, will replace the manuscript version online.

\section{MEDIA COVERAGE:}

If your internal Public Relations (PR) office plans a news release or other PR-related activity for this paper, please send an e-mail to lauren.riley@aacr.org. A member of the AACR Communications Team will liaise with your internal PR office to make sure embargo policies are followed.

\section{ARCHIVING MANDATES:}

AACR journals can help you to fulfill many funders' mandates to archive your accepted manuscript by depositing your manuscript for you in PubMed Central (PMC) or Europe PubMed Central (Europe PMC). If your research is funded by the National Institutes of Health, Howard Hughes Medical Institute, Wellcome Trust, Cancer Research UK, and/or the Medical Research Council, AND IF YOU SELECTED THE ARCHIVING OPTION WHEN YOU SUBMITTED YOUR PAPER, you need to do nothing else. 
If you did not select the archiving option and now wish to make use of the AACR journals' archiving service for authors, please contact the CANCER RESEARCH Editorial Office (cancerres@aacr.org) for assistance.

More information about AACR archiving, copyright, and permission policies is available at http://www.aacrjournals.org/site/InstrAuthors/ifora.xhtm|\#mandatesassist.

Again, congratulations on the acceptance of your manuscript for publication in CANCER RESEARCH, a member of the AACR family of journals that publishes some of the most significant research in the field. As an author advancing the science in cancer research, you may want to join the nearly 30,000 experts in the field who already take advantage of AACR's many benefits of membership. These include substantially reduced fees for meeting registrations and journal subscriptions, as well as excellent opportunities to foster important relationships and collaborations with colleagues internationally. For further information or to download a membership application, please visit http://www.aacr.org/Membership/Pages/become-amember 02B2B7.aspx.

Sincerely,

Laura E. Benjamin, PhD

Deputy/Senior Editor

Cancer Research 
MiR-9 and miR-200 regulate PDGFRß-mediated endothelial differentiation of tumor cells in triple negative breast cancer

Elvira D'Ippolito ${ }^{1 *}$, Ilaria Plantamura ${ }^{1 *}$, Lucia Bongiovanni ${ }^{2}$, Patrizia Casalini ${ }^{3}$, Sara Baroni ${ }^{1}$, Claudia Piovan ${ }^{1}$, Rosaria Orlandi ${ }^{3}$, Ambra V Gualeni ${ }^{4}$, Annunziata Gloghini ${ }^{4}$, Anna Rossini ${ }^{5}$, Sara Cresta $^{5}$, Anna Tessari ${ }^{5}$, Filippo De Braud ${ }^{5}$, Gianpiero Di Leva ${ }^{6}$, Claudio Tripodo ${ }^{2}$ and Marilena V Iorio ${ }^{1}$

${ }^{1}$ Start Up Unit, Department of Experimental Oncology and Molecular Medicine, Fondazione IRCCS Istituto Nazionale dei Tumori, Milan, 20133, Italy; ${ }^{2}$ Tumor Immunology Unit, Department of Health Sciences, University of Palermo, Palermo, 90133, Italy; ${ }^{3}$ Molecular Targeting Unit, Department of Experimental Oncology and Molecular Medicine, Fondazione IRCCS Istituto Nazionale dei Tumori, Milan, 20133, Italy; ${ }^{4}$ Department of Diagnostic Pathology and Laboratory Medicine, Fondazione IRCCS Istituto Nazionale dei Tumori, Milan, 20133, Italy; ${ }^{5}$ Department of Medical Oncology, Fondazione IRCCS Istituto Nazionale dei Tumori, Milan, 20133, Italy; ${ }^{6}$ Environment \& Life Sciences, University of Salford, Salford, M5 4WT, UK. Manchester, M13 9PL , United Kingdom.

*these authors equally contributed to this work

Running title: miRNAs in PDGFR $\beta$-mediated vasculogenesis of TNBC Keywords: breast cancer/microRNAs/PDGFR $\beta /$ vasculogenesis/EMT

Financial support. Dr. Iorio is supported by a START UP AIRC grant (N 11699) and by a Young Investigator grant from Italian Ministry of Health.

Corresponding author: Marilena V. Iorio, Start Up Unit, Dept. of Experimental Oncology and Molecular Medicine, Fondazione IRCCS Istituto Nazionale dei Tumori, Via Amadeo 42, 20133 Milan, Italy. Phone: +39-02-23905134/26. Fax: +39-02-23902692.

Email address: marilena.iorio@istitutotumori.mi.it

Conflicts of interest: None of the authors have competing interests to declare.

Word count: 5500

Number of figures and tables: 6 figures and 1 table 


\begin{abstract}
Organization of cancer cells into endothelial-like cell lined structures to support neovascularization and fuel solid tumors is a hallmark of progression and poor outcome. In triple negative breast cancer (TNBC), PDGFR $\beta$ has been identified as a key player of this process and is considered a promising target for breast cancer therapy. Thus, we aimed at investigating the role of microRNAs as therapeutic approach to inhibit PDGFR $\beta$-mediated vasculogenic properties of TNBC, focusing on miR-9 and miR-200.
\end{abstract}

In MDA-MB-231 and MDA-MB-157 TNBC cell lines, miR-9 and miR-200 promoted or inhibited, respectively, the formation of vascular-like structures in vitro. Induction of endogenous miR-9 expression, upon ligand-dependent stimulation of PDGFR $\beta$ signaling, promoted significant vascular-sprouting of TNBC cells in part by direct repression of STARD13. Conversely, ectopic expression of miR-200 inhibited this sprouting by indirectly reducing the protein levels of PDGFR $\beta$ through the direct suppression of ZEB1.

Notably, in vivo miR-9 inhibition or miR-200c restoration, through either the generation of MDAMB-231 stable clones or peritumoral delivery in MDA-MB-231 xenografted mice, strongly decreased the number of vascular lacunae. Finally, immunohistochemistry and immunofluorescence analyses in TNBC specimens indicated that PDGFR $\beta$ expression marked tumor cells engaged in vascular lacunae.

In conclusion, our results demonstrate that miR-9 and miR-200 play opposite roles in the regulation of the vasculogenic ability of TNBC, acting as facilitator and suppressor of PDGFR $\beta$, respectively. Moreover, our data support the possibility to therapeutically exploit miR-9 and miR200 to inhibit the process of vascular lacunae formation in TNBC. 


\section{Introduction}

Tumor vascularization is a fundamental step in solid tumor progression. The idea that this process only relies on the sprouting of existing endothelial angiogenic vessels has been gradually replaced by the evidence that tumor vasculature is orchestrated by different pathways of vasculogenesis. Indeed, the contribution of neoplastic cells is increasingly relevant (1). More importantly, the presence of non-canonical mechanisms of tumor-mediated vascularization (e.g. vascular mimicry, tumor to endothelial-like cell differentiation, vessel co-option) is particular to more aggressive cancers and has been associated with tumor progression and decreased survival rate (1).

The differentiation of tumor cells in endothelial-like cells was reported in angiogenic vessels of aggressive cancers, where neoplastic cells acquired the expression of endothelial markers (e.g. CD31 and CD34) and participated in the formation of functional vascular-like structures $(2 ; 3)$. We recently described the presence of vascular lacunae lined by a mosaic of normal endothelial cells and CD31-expressing tumor cells in human breast cancer (4). Among the different breast cancer subtypes, we reported that the endothelial differentiation ability was most prominent in triple negative breast cancer (TNBC), the most aggressive and undifferentiated subtype $(5 ; 6)$, where the presence of vascular lacunae associated with poor clinical outcome. Moreover, treatment with multi-target tyrosine kinase inhibitors (TKIs) reduced the formation of these structures, nominating Platelet-Derived Growth Factor Receptor Beta (PDGFR $\beta$ ) as an important player in this alternative mechanism of vascularization (4). Interestingly, PDGFR signaling has been under investigation as a promising target for breast cancer therapy due to its involvement in crucial steps of progression such as angiogenesis, epithelial to mesenchymal transition (EMT) and metastasis (7). However, clinical trials with TKIs against this receptor have not produced satisfactory results (7). 
MicroRNAs (miRNAs) are small non-coding RNA molecules involved in gene regulation that are often deregulated in human cancer (8). Interestingly, a negative regulatory loop involving miR-9 and PDGFR $\beta$ has been described in a cardiomyocyte model (9). In this context, ligand-dependent stimulation of PDGFR $\beta$ triggers activation of the downstream signaling pathways. PDGFR signaling induces the expression of miR-9, which acts to inhibit PDGFR $\beta$ activation by targeting the mRNA encoding this receptor. Together with the internalization and degradation of PDGFR $\beta$ upon stimulation, this provides a mechanism by which signaling through this receptor may be temporally limited. High levels of miR-9 have been associated with pro-metastatic function in human breast cancer (10), as well as with the acquisition of a mesenchymal and aggressive phenotype (11). On the other hand, members of the miR-200 family (five miRNAs organized in two clusters: miR-200a/b/429 and miR-200c/141) are well-known negative regulators of EMT due to their direct targeting of several transcriptional factors implicated in the transition, such as ZEB1/2 and Snail (12). Interestingly the EMT-associated transcriptional factors FOXQ1 and TWIST1 can induce PDGFR expression (13). Conversely, PDGFR $\beta$ activation with PDGF-DD ligand mediates EMT through the induction of ZEB1 and consequent suppression of miR-200 (14).

Here, we investigated the role of miR-9 and miR-200 in the PDGFR $\beta$-mediated vasculogenic properties of TNBC, raising the possibility of a new miRNA-based therapeutic approach to impair the phenomenon of vasculogenesis in TNBC. 


\section{Materials and Methods}

\section{Patients and samples}

Human tissues were selected from the archives of the Fondazione IRCCS Istituto Nazionale dei Tumori of Milan (INT) from patients with invasive breast cancer and naïve of neoadjuvant treatment. The first set included 78 formalin-fixed paraffin embedded (FFPE) breast cancers collected from 2003 to 2004 (29 luminal, 32 human epidermal growth factor receptor 2 positive (HER2+) and 17 TNBCs). The second set consisted of 85 FFPE TNBC, resected between 2002 and 2006 (baseline characteristics of the two cohorts in Supplementary Table S1). Histologic subtype and grade were determined according to WHO classification and Nottingham histologic grading system, respectively. Immunohistochemistry (IHC) classification was assigned following the 2009

St. Gallen Consensus guidelines for estrogen receptor (ER) and progesterone receptor (PgR) markers, whereas HER2 was scored according to ASCO/CAP 2013 guidelines. An informed consent was obtained from all patients. All procedures were carried out in accordance with the Helsinki Declaration (World Medical Association, 2013) and the study was conducted only after approval from the Institutional Review Board and the Independent Ethical Committee.

\section{Cell cultures, plasmids and treatments}

Human TNBC cell lines MDA-MB-231, MDA-MB-157, MDA-MB-468 and HCC1937, and embryonic kidney HEK293 were purchased from ATCC (Rockville, MD). SUM149 and SUM159 TNBC cell lines were purchased from Asterand Bioscience (Detroit, MI). All cell lines were obtained between 2000 and 2010, authenticated once a year (last verification on November 2015) using the short tandem repeat profiling method in our Institute facility, and propagated in the suggested media within six months of thawing from stocks.

Clones stably expressing miR-9 inhibitor and miR-200c precursor were generated from the MDA-MB-231 cell line after transfection with pEZX-AM01 and pEZX-AM04 plasmids (GeneCopoeia, 
Rockville, MD), respectively, using Lipofectamine 3000 transfection reagent (Life Technologies, Carlsbad, CA). Cells were cultured in RPMI 1610 medium with $10 \%$ FBS, 1 mM L-glutamine and $0.75 \mu \mathrm{g} / \mathrm{ml}$ (miR-9 clones) or $0.5 \mu \mathrm{g} / \mathrm{ml}$ (miR-200c clones) puromycin.

For PDGFRß stimulation, cells were treated with $50 \mathrm{ng} / \mathrm{ml}$ PDGF-BB (Peprotech, Rocky Hill, NJ) in serum-free medium and maintained in serum-free medium.

\section{MiRNA and siRNA transfection}

MiRNA over-expression was achieved by transfection with human miRNA precursors (Life Technologies) and verified by qRT-PCR. MiRNA silencing was obtained by transfection with locked nucleic acid (LNA)-based inhibitors (Exiqon, Vedbaek, DK) and validated by reporter assay. For gene knock down, specific siRNAs (Life Technologies) were used. Cells were incubated with 100 nM miRNA precursors, LNAs or siRNAs complexed with Lipofectamine RNAi Max transfection reagent (Life Technologies) according to manufacturer's instructions.

\section{RNA extraction and quantitative RT-PCR}

Total RNA was extracted from cell lines with Qiazol reagent (Qiagen, Valencia, CA). For gene and miRNA quantification, cDNA was synthesized from $1 \mu \mathrm{g}$ and $100 \mathrm{ng}$ of RNA with SuperScript III Reverse Transcriptase and TaqMan MiRNA Reverse Trascription Kit (Life Technologies), respectively. QRT-PCR was performed using TaqMan assays for human ZEB1, GAPDH, miR-9-5p, miR-200b-3p, miR-200c-3p and RNU44 (Life Technologies) or custom primers for PDGFRB and GAPDH with SYBR Green technology (Life Technologies). From FFPE breast cancer tissues, total RNA was extracted with miRNeasy mini kit (Qiagen) and assessed for quality via Bioanalyzer. $20 \mathrm{ng}$ of RNA were reverse transcribed using miRCURY LNA Universal RT miRNA PCR system (Exiqon) and qRT-PCR was performed in triplicate using Exiqon assays for human miR-9-5p, miR-200c-3p, RNU44 and RNU48. Gene and miRNA levels were normalized to the endogenous control RNU44, $R N U 48$ or GAPDH and the relative expression was calculated using the comparative $2^{-\triangle \mathrm{Ct}}$ method. 
Primers: GAPDH Fw: 5'-ATTCCACCCATGGCAAATTC-3'; GAPDH Rv: 5'-AGCATCGCCCCACTTGATT3'; PDGFRB Fw: 5'-AGCGCTGGCGAAATCG-3'; PDGFR6 Rv: 5'-TGACACTGGTTCGCGTGAA-3'

\section{Western blot}

Total protein lysates were extracted with lysis buffer (1\% Triton, 50nM Tris, $15 \mathrm{mM} \mathrm{NaCl})$ supplemented with protease inhibitors (Sigma-Aldrich, St. Louise, MO). The following primary antibodies were used: rabbit anti-human IgG antibodies against STARD13 (1:500, sc-67843), PDGFRß (1:500, sc-432), E-cadherin (1:500, sc7870) (Santa Cruz, Dallax, TX); mouse anti-human IgG antibody against Vinculin (1:1000, V9131, Sigma-Aldrich); mouse horseradish peroxidaseconjugated anti-human IgG anti- $\beta$ actin (1:1000, A3854, Sigma-Aldrich). Proteins were visualized by enhanced chemiluminescence detection system (Sigma-Aldrich). Quantification was performed by Quantity One 4.6.6 software (Bio-Rad, Hercules, CA).

\section{Reporter assay}

$8 \times 10^{4}$ cells were seeded in 24-well plates and co-trasfected with 500 ng pMiR-9-5p-Luc reporter vector (Signosis, Santa Clara, CA) and 50 ng phRL-SV40 control vector (Renilla) (Promega, Madison, WI) using Lipofectamine 3000. After $24 \mathrm{~h}$, Firefly and Renilla luciferase activities were measured by Dual-Luciferase Reporter Assay System (Promega).

\section{Tube formation assay}

96-well plates were coated with growth factor reduced Matrigel (Corning, New York, NY) and tube formation assay was performed and quantified as previously reported (4).

\section{Dual-luciferase reporter assay}

The full length human 3'UTR of STARD13 was cloned in pmirGLO Dual-Luciferase miRNA Target Expression vector (Promega) (pmiR-3'UTRWT). This construct was used to generate plasmids carrying the mutated forms of STARD13 3'UTR, modified in each of the two predicted miR-9 binding sites (pmiR-3'UTRMut1 and pmiR-3'UTRMut2), using QuikChange II XL Site-Directed 
Mutagenesis Kit (Agilent, Santa Clara, CA). $2 \times 10^{5}$ HEK293 cells were seeded in 12-well plates and co-transfected with 500 ng pmiR-3'UTR (WT, Mut1 or Mut2) and 100 nM of either miR-9-5p precursor or negative control using Lipofectamine 3000. After $24 \mathrm{~h}$ Firefly and Renilla luciferase activities were measured as described above.

\section{In silico analyses}

Level 3 TCGA data from mRNA-seq and miR-seq of breast cancer samples were used. To define tumor subtype we used ER, PgR and HER2 IHC status reported in the clinical information file.

Profiling data of 175 nitrogen-frozen breast cancer tissues had been deposited by Huang and colleagues in the Gene Expression Omnibus data repository (GEO) with accession number GSE59590 (15) . For the Italian subset, differentially expressed genes between luminal and basal breast cancers were identified by linear modeling as implemented in the limma package (16). In silico prediction of miRNA targets was performed with 6 algorithms simultaneously (DIANAMicroTCDS, miRanda, miRDB, PITA, RNA22, TargetScan v6.2) (17) using the HUGO gene symbol as common identifier.

\section{In vivo experiments}

Orthotopic breast tumors were generated by implantation of $5 \times 10^{6}$ cells resuspended in a 1:1 mixture of PBS and matrigel (Corning) in the mammary fat pad (m.f.p.) of 8 week-old female SCID mice (Charles Rivers, Wilmington, MA). For stable clones, tumors were monitored and harvested before necrosis. For in vivo miRNA modulation, when MDA-MB-231 tumors reached a volume of approximately $50 \mathrm{~mm}^{3}$, mice were treated with $20 \mu \mathrm{g}$ miRNA-based drugs (Tema Ricerca, Bologna, IT) administered 5 times, every 3-4 days, by peritumoral injection. MiR-9 inhibitor or control were delivered as naked oligonucleotides, whereas miR-200c mimic or cel-miR-67 control were formulated with MaxSuppressor In Vivo RNA-LANCEr II (BIOO Scientific, Austin, TX), according to 
manufacturer's instructions. All animal experiments were approved by the Ethics Committee for Animal Experimentation of INT.

\section{Immunohistochemical analysis}

FFPE xenograft tumor sections were unmasked using Novocastra Epitope Retrieval Solutions $\mathrm{pH} 6$ and $\mathrm{pH} 9$ and incubated $1 \mathrm{~h}$ with the following primary antibodies: mouse anti-human CD31 (1:50, 1A10, Leica Biosystems, Wetzlar, DE); mouse anti-human AREB6 (ZEB1) (1:150, 3G6, Abcam, Cambridge, UK), at room temperature.

FFPE human specimens were treated with citrate solution to unmask the antigen and then incubated $1 \mathrm{~h}$ with rabbit anti-human PDGFR $(1: 200, \mathrm{Y} 92, \mathrm{Abcam})$ at room temperature.

\section{Immunofluorescence}

FFPE human sections were incubated with the following primary antibodies: mouse anti-human CD31 (1:50 pH6, 1A10, Leica Biosystems), rabbit anti-human PDGFRß (1:250 pH6, Y92, Abcam) and mouse anti-human p53 (1:800 pH6, DO-7, Leica Biosystems). The following secondary antibodies were used: Alexa Fluor 350-conjugated goat anti-mouse, Alexa Fluor 488-conjugated goat antirabbit, Alexa Fluor 568-conjugated goat anti-mouse (Life Technologies).

\section{MiRNA in situ hybridization}

MiRNA in situ hybridization (ISH) was performed as previously described (18). Tumor sections were hybridized with double-DIG-LNA probes for miR-21, miR-9, miR-200c and scrambled miR (Exiqon), according to manufacturer's instructions.

\section{Statistics}

For two group comparison, multiple group comparison and correlation analyses, the probability value was calculated, respectively, with either an unpaired two-tailed Student's $t$-test or nonparametric Mann-Whitney test, non-parametric Kruskal-Wallis test or moderated t-test adjusted for multiple testing by the false discovery rate (FDR) on limma package in $\mathrm{R}$, and Pearson test using 
Graph Pad Prism 5 software (GraphPad software Inc., San Diego, CA). Statistical significance of association analyses and differences in survival Kaplan-Meier curves were tested with chi-square and Wilcoxon test, respectively, using SAS software (SAS Institute Inc., Cary, NC). $p \leq 0.05$ was considered significant.

Data are expressed as mean \pm standard deviation of three independent experiments, unless otherwise specified in the figure legends. 


\section{Results}

\section{MiR-9 mediates PDGFRß-induced tube formation ability}

Among a panel of TNBC cell lines, we selected MDA-MB-231 and MDA-MB-157 as suitable models based on their described ability to generate vascular-like structures in vitro (4) and on the opposite expression of miR-9 (Supplementary Fig. S1A). We first confirmed the previously described negative regulatory loop existing between miR-9 and PDGFR $\beta$ (9). Specifically, PDGFR $\beta$ protein levels were reduced upon over-expression of miR-9 in MDA-MB-231 cells, and increased following inhibition of this miRNA in MDA-MB-157 cells (Supplementary Fig. S1B-C), corroborating the targeting of the receptor by miR-9. Further reporting the previously described regulatory relationship between PDGFR $\beta$ and miR-9 (9), activation of PDGFR $\beta$ with PDGF-BB ligand increased the relative expression of miR-9 in both TNBC models (Fig. 1A).

We thus investigated the functional effect of miR-9 in tube formation capability. MDA-MB-231 cells transfected with miR-9 precursor acquired a higher ability to form vascular-like structures than control cells, whereas silencing of miR-9 in MDA-MB-157 cells resulted in a strong impairment of the same phenomenon (Fig. 1B). These data suggest that miR-9 could contribute to PDGFR $\beta$-regulated vasculogenesis. To strengthen these findings, MDA-MB-157 cells were transfected with either LNA-miR-9 or a scrambled oligonucleotide, and PDGF-BB-treated cells were compared to control cells. We observed that the advantage in loop formation ability gained with the treatment with PDGF-BB was almost completely abrogated by concomitant knock down of miR-9 (Fig. 1C).

In summary, miR-9 enhances the ability of TNBC cells to generate vascular-like structures in response to PDGFRß in vitro.

MiR-9 is overexpressed in TNBC and associates with poor prognosis in breast cancer 
Considering the ability of miR-9 to enhance vasculogenic properties and the stronger capability of TNBC to generate vascular lacunae than luminal and HER2+ carcinomas (4), we investigated whether miR-9 levels vary among the different breast cancer subtypes. In a cohort of 78 breast cancer tissues (set 1), we observed higher expression of miR-9 in TNBC than luminal and HER2+ subtypes, a finding that was further validated in the TCGA public dataset (Supplementary Fig. S2AB). Moreover, we observed higher miR-9 levels in tumors with high histological grade $(p<0.0001)$ and negative hormonal status (ER $p=0.0088, \operatorname{PgR} p=0.0007$ ) (Supplementary Table S2). Finally, breast cancer patients were stratified according to miR-9 median expression. Kaplan-Meier survival analysis showed that higher miR-9 levels significantly associated with poor prognosis in terms of both disease-free survival (DFS) and distant-metastasis free survival (DMFS) $(p=0.0077$ and $p=0.0121$, respectively) (Supplementary Fig. S2C).

\section{MiR-9 regulates TNBC vasculogenic properties through STARD13 targeting}

To identify additional miR-9 targets that might be able to explain its effect on the vasculogenic properties of TNBC, we took advantage of deposited gene profiling data of Italian and Chinese breast cancer patients (15). Tumors were stratified as luminal, HER2+, basal, or normal-like subtype, according to intrinsic gene expression (19). Considering the overlapping expression profiles of basal and TNBC subtypes (6), we analyzed 13 basal versus 80 luminal tumors belonging to the Italian cohort of the dataset. After validating higher miR-9 expression in the basal subtype (Fig. 1D), we merged genes down-regulated in basal versus luminal breast cancers with miR-9 targets predicted by at least 3 out of 6 prediction tools. One of the most differentially expressed genes in our dataset, STARD13 (StAR-related lipid transfer domain containing 13 - a Rho-GAPaseactivating protein), met these criteria (Supplementary Table S3). Given that down-regulation of this gene has been associated with increased motility and invasion (20), we examined STARD13 as a putative mediator of miR-9 effect on TNBC vasculogenesis. 
We first validated STARD13 as miR-9 target by assessing changes in the relative expression of this protein following miR-9 transfection. Consistent with STARD13 being a downstream target of miR-9 activity, we observed a slight reduction in both TNBC cell lines following this transfection (Fig. 1E). Furthermore, siRNA-mediated knock down of STARD13 accelerated the formation of vascular-like structures, phenocopying the effects resulting from overexpression of miR-9 (Fig. 1F). Finally, miR-9-mediated suppression of luciferase activity in HEK293 cells co-transfected with a reporter fused to the 3' UTR of STARD13 (pmiR-3'UTRWT) was abrogated by mutating at least one of the two miR-9 binding sites (Fig. 1G).

In conclusion, we show that STARD13 is directly targeted by miR-9, and this targeting comprises one of the mechanisms exploited by the miRNA to modulate the vasculogenic properties of TNBC cells.

\section{MiR-200 family inhibits tube formation ability through PDGFR $\beta$ repression}

We next evaluated the basal levels of miR-200 in the same panel of TNBC cell lines, using miR$200 \mathrm{~b}$ and miR-200c as representative members of each of the two clusters comprising the miR200 family. We found that both miRNAs were strongly down-regulated in the mesenchymal and stem-like Basal B compared to the more epithelial Basal A subgroup (Supplementary Fig. S3A). In the selected MDA-MB-231 and MDA-MB-157 cell lines, ectopic expression of both miR-200 members not only strongly impaired tube formation ability (Fig. 2A and Supplementary Fig. S3B), but also restored E-cadherin expression and, more interestingly, led to an important reduction in PDGFR $\beta$ protein levels (Fig. 2B).

Since PDGFR $\beta$ is not a predicted target of miR-200, we investigated how this family could regulate the expression of the receptor. The induction of E-cadherin after miR-200 restoration is a hallmark of the suppression of ZEB1, which is a known target of miR-200 (21). This observation, combined with the possible role of ZEB1/PDGFR $\beta$ cross-talk in the maintenance of a post-EMT 
phenotype $(13 ; 22)$, raised the hypothesis that miR-200 could indirectly repress PDGFR $\beta$ through ZEB1 targeting. For this reason, we first investigated the role of ZEB1 in in vitro loop formation. As expected, knockdown of ZEB1 reduced the ability of MDA-MB-231 cells to form vascular-like channels (Fig. 2C). Moreover, ZEB1 silencing significantly reduced PDGFR $\beta$ at both the mRNA and protein level in both MDA-MB-231 and MDA-MB-157 cell lines (Fig. 2D and Supplementary Fig. S3C-E). Finally, we found a strong positive correlation between mRNA levels of ZEB1 and PDGFRB in the TNBC subset of the TCGA dataset ( $p<0.001, r=0.8098)$ (Fig. 2E).

We concluded that members of the miR-200 family inhibit the tube formation ability of TNBC cell lines through the suppression of PDGFR $\beta$. Furthermore, the regulation of the receptor by miR200 strongly relies on the targeting of ZEB1.

\section{MiR-9 and miR-200c regulate vascular lacunae formation in vivo}

To validate the effect of the miRNAs of interest on vasculogenic properties in vivo, we first generated MDA-MB-231 clones for the stable inhibition of miR-9 (sponge miR-9) or overexpression of miR-200 (miR-200c) and the corresponding controls (sponge miR-control and vecmiR, respectively), selecting miR-200c as representative member of the family (Fig. $3 A$ ). The expanded clonal populations were implanted in the mammary fat pad of female SCID mice and monitored until the time of sacrifice. MiR-9 knock down did not lead to significant differences in tumor volume, whereas miR-200c tumor-bearing mice exhibited a strong reduction in tumor growth compared to control group (Fig. 3B). To assess the effect on vasculogenic properties, tumors were analyzed for the presence of vascular lacunae, identified as CD31-positive blood vessels with CD31-positive tumor cells lining the vascular structure. Qualitative analysis first revealed the presence of less structured vascular lacunae following both inhibition of miR-9 and over-expression of miR-200c in comparison with control groups (Fig. 3C). In addition, we observed 
a significant reduction in the number of CD31-positive vascular lacunae compared to the corresponding controls (Fig. 3D).

We then adopted the MDA-MB-231 cell line to perform in vivo miRNA delivery experiments on tumors orthotopically xenografted in SCID mice. MiR-9 inhibitor or miR-200c mimic, and respective controls, were delivered by peritumoral injection. Consistently with the results obtained with stable clones, mice treated with miR-200c mimic but not those treated with miR-9 inhibitor showed a significant reduction of tumor volume (Fig. 4A). Moreover, the analysis of CD31-positive vascular lacunae highlighted a significant qualitative and quantitative reduction of the vasculogenic capability in treated versus untreated xenografts (Fig. 4B-C). Finally, to verify the efficiency of the delivery, histological sections were analyzed for the expression of the EMTassociated transcription factor ZEB1. Examination of these sections revealed an overall decrease in treated samples in comparison with controls (Fig. 4D).

\section{PDGFR $\beta$ identifies vascular lacunae in TNBC tissues}

Having assessed the functional role of PDGFR $\beta$ in mediating vasculogenic properties, we evaluated the ability of the receptor to identify vascular lacunae in TNBC human tissues, as previously reported for the CD31 marker (4).

In the TNBC cohort (set 2), IHC analysis revealed a variable membrane and cytoplasmic staining of PDGFR $\beta$ in both stromal and epithelial structures. As expected, perivascular fibroblasts and pericytes associated with blood vessels were PDGFR $\beta$ positive; intratumoral stromal axes surrounding nests of neoplastic cells showed a moderate-to-intense positivity for the receptor. Finally, neoplastic cells showed a variable reactivity, ranging from absence of signal to a strong cytoplasmic and membrane staining (Supplementary Fig. S4A-B). More importantly, as shown for CD31 marker, also PDGFR $\beta$ was able to identify tumor cells physically engaged in the formation of vascular lacunae (Fig. 5A). To strengthen this evidence, we performed a triple-marker 
immunofluorescence (IF) analysis, observing foci of co-localization of PDGFR $\beta$ and CD31 in tumor cells, identified by positive reactivity to p53 staining (Fig. 5B).

TNBC tumors were then divided according to the presence (PDGFR $\beta+$ ) or absence (PDGFR $\beta-$ ) of PDGFR $\beta$ staining associated to tumor cells either engaged in vascular-like structures or organized in tumor nests (Table 1). We first found a strong association between the presence of CD31+ and PDGFR $\beta+$ vascular lacunae $(p=0.001)$. In addition, the presence of PDGFR $\beta+$ vascular lacunae strongly associated also with the presence of PDGFR $\beta+$ tumor nests $(p<0.001)$. As a consequence, 79,3\% of tumors with PDGFRß + tumor nests showed CD31+ vascular lacunae (Table 1). Interestingly, PDGFR $\beta$ expression in both tumor nests and vascular lacunae associated with high histological grade $(p=0.095)$. We did not observe any significant association with other clinicopathologic parameters (Table 1).

In light of the opposite behavior of miR-9 and miR-200 family in regulating PDGFR $\beta$-mediated vasculogenic properties, we finally evaluated the association between the expression levels of these miRNAs and the receptor status in the TNBC cohort. We found that high miR-200c levels negatively associated with the presence of PDGFR $\beta+$ vascular lacunae $(p=0.0444)$ and established a trend of negative association with PDGFRß+ tumor nests (Supplementary Table S4).

Finally, we qualitatively assessed the tumor distribution of miR-9 and miR-200c in TNBC by ISH. Interestingly, miR-200c expression was mainly detected in tumor cells, whereas absence of signal was observed in the stromal compartment and immune infiltrate (Fig. 5C). Conversely, we were not able to detect miR-9, probably because miR-9 expression was below the ISH detection threshold. MiR-21 and scrambled miR were used as positive and as negative controls, respectively (Supplementary Fig. S4C). 


\section{Discussion}

Our findings indicate that miR-9 and miR-200 play opposite roles in regards to PDGFR $\beta$ mediated vasculogenic properties of TNBC. Further, we demonstrate that PDGFR $\beta$ staining identifies tumor cells physically engaged in the formation of vascular lacunae.

MiR-9 has a well-recognized pro-metastatic function in human breast cancer $(10 ; 23)$, and it has been more recently associated with poor prognosis, EMT and stemness features (11). Consistently, we found that higher miR-9 expression identified breast cancer patients with poor prognosis and associated with high histological grade. Moreover, miR-9 levels were higher in TNBC than in luminal and HER2+ subtypes, in accordance with the negative association with hormonal status; ER can in fact mediate the epigenetic silencing of miR-9 (24), strengthening the preferential expression of this miRNA in more aggressive phenotypes.

The pro-tumoral effects of miR-9 (e.g. migration, invasion, EMT) resemble the features mediated by PDGFR $\beta(25 ; 26)$. We showed indeed that miR-9 itself was able to enhance vasculogenic properties both in vitro and in vivo, resembling the addiction of TNBC to PDGFR $\beta$ signaling for the generation of vascular lacunae (4). Moreover, miR-9 expression was induced upon PDGFR $\beta$ activation, suggesting that this miRNA acts as downstream effector of the receptor signaling. Indeed, PDGF-BB-mediated induction of tube formation capability was abrogated by concomitant inhibition of miR-9, thus demonstrating that this miRNA is indeed crucial for PDGFR $\beta$ mediated enhancement of vasculogenesis.

We identified STARD13 as a new miR-9 direct target. Despite the improvement of vasculogenic properties of TNBC cell lines after STARD13 silencing, we only partially phenocopied the effect of miR-9 over-expression. Nevertheless, it is known that miRNAs explicate their action through the targeting of multiple gene products; thus, we would need a more extensive study on miR-9 targets to fully recapitulate its effect on vasculogenesis. 
Even though our data support the connection between miR-9 and PDGFR 3 , in the TNBC set we did not observe a significant association between miR-9 expression and the presence of either vascular lacunae or tumor nests positive for the receptor. However, unlike miR-200c, we were not able to reliably assess miR-9 distribution within the tumor microenvironment by ISH. Since we cannot exclude other non-tumoral sources of this miRNA, miR-9 levels detected by qRT-PCR might not mirror the expression in tumor cells.

The miR-200 family regulates fundamental steps of breast cancer progression, although its role in tumorigenesis still appears contradictory. This miRNA family, in fact, inhibits tumor proliferation, migration, invasion, stemness and contributes to overcome resistance to standard therapies (21;27-31), but its expression has been associated with breast cancer aggressiveness (32;33). Moreover, miR-200 can either increase or reduce the metastatic potential in different TNBC models (34;35). However, miR-200 expression is very heterogeneous within TNBC and epigenetically suppressed in undifferentiated and plastic phenotypes (36;37). We confirmed that cell lines with mesenchymal and stem-like features (Basal B) exhibited a more significant loss in the expression of miR-200 than cells with epithelial features (Basal A); in addition, in vivo restoration of miR-200c in Basal B models strongly inhibited tumor growth, corroborating its tumor suppressor role. More interestingly, we described a new antitumor activity of this family consisting in the impairment of tumor cell-mediated vascularization, which is exerted through the suppression of PDGFR $\beta$. Furthermore, the negative association between miR-200c expression and PDGFR $\beta$-positive vascular lacunae supported the existence of this anti-correlation also in human TNBC. Finally, the predominant expression of miR-200c in tumor cells focused the miR200/PDGFR $\beta$ cross-talk in the tumor compartment.

PDGFR $\beta$ is not, however, predicted as direct target of miR-200. Our data strongly suggest that ZEB1, one of the main transcriptional factors triggering EMT, is the link between miR-200 and 
PDGFR $\beta$. Consistently, a higher PDGFR $\beta$ expression has been reported in breast cancers with a more prominent mesenchymal phenotype (38).

In vivo experiments support the effect of miR-9 knock down and miR-200 restoration in the impairment of vascular lacunae formation. The approach of miRNA-based drug delivery raised two interesting considerations. First, miRNA treatments induced ZEB1 suppression in xenograft tumors, highlighting the ability of miR-9 and miR-200 to regulate not only tumor-mediated vasculogenesis but also, more generally, EMT. Interestingly, Twist1, another key transcriptional factor that triggers EMT, was recently reported to induce the endothelial transition of tumor cells (39). Moreover, this approach represents a proof of concept for exploiting miR-9 and miR-200 as therapeutic tools to affect tumor vascularization. The existence of pathways that act as alternatives to the canonical process of sprouting angiogenesis are proposed to mediate resistance to conventional anti-angiogenic therapies (40). Intriguingly, miR-9 and miR-200 can directly induce or inhibit, respectively, also the canonical angiogenesis $(41 ; 42)$, supporting their modulation as a new approach to simultaneously target different aspects of tumor vascularization.

Finally, our analyses show that PDGFRß-expressing tumor cells identify vascular lacunae in human TNBC. More interestingly, the only detection of tumor nests positive for PDGFR $\beta$ strongly indicates the presence of vascular lacunae, suggesting that the IHC evaluation of the receptor could be useful in the identification of patients whose tumor displays this aggressive phenotype. However, the absence of PDGFR $\beta$ signal does not exclude the presence of tumor-mediated vascularization, as highlighted by the existence of PDGFRß-negative/CD31-positive vascular lacunae.

In conclusion, we describe a new role of miR-9 and miR-200 in the biology of TNBC. The tight relation between PDGFRß-miR-9 axis and EMT, with concomitant down-regulation of miR-200, generates a favorable environment for the sustenance of tumor-mediated vasculogenesis, which 
can be impaired by treatments with miR-9 inhibitors or miR-200 mimics (Fig. 6). A deeper investigation of miR-9 and miR-200 in these multiple canonical and non canonical pathways of vasculogenesis becomes indeed fundamental.

\section{Acknowledgements}

Thanks to Dr. Elda Tagliabue for the useful scientific discussion; to Dr. Sandra Romero-Cordoba for in silico analysis on public TCGA dataset; to Dr. Manuela Campiglio for providing clinicalpathological information on TNBC dataset; to Dr. Cristina Ghirelli for help with cell cultures and to Laura Mameli for reference editing. 
References

(1) Cao Z, Shang B, Zhang G, Miele L, Sarkar FH, Wang Z, et al. Tumor cell-mediated neovascularization and lymphangiogenesis contrive tumor progression and cancer metastasis. Biochim Biophys Acta 2013;1836:273-86.

(2) Ricci-Vitiani L, Pallini R, Biffoni M, Todaro M, Invernici G, Cenci T, et al. Tumour vascularization via endothelial differentiation of glioblastoma stem-like cells. Nature 2010;468:824-8.

(3) Soda $Y$, Marumoto T, Friedmann-Morvinski D, Soda M, Liu F, Michiue H, et al. Transdifferentiation of glioblastoma cells into vascular endothelial cells. Proc Natl Acad Sci U S A 2011;108:4274-80.

(4) Plantamura I, Casalini P, Dugnani E, Sasso M, D'Ippolito E, Tortoreto M, et al. PDGFR $\beta$ and FGFR2 mediate endothelial cell differentiation capability of triple negative breast carcinoma cells. Mol Oncol 2014;8:968-81.

(5) Dent R, Trudeau M, Pritchard KI, Hanna WM, Kahn HK, Sawka CA, et al. Triple-negative breast cancer: clinical features and patterns of recurrence. Clin Cancer Res 2007;13:4429-34.

(6) Carey L, Winer E, Viale G, Cameron D, Gianni L. Triple-negative breast cancer: disease entity or title of convenience? Nat Rev Clin Oncol 2010;7:683-92.

(7) Criscitiello C, Gelao L, Viale G, Esposito A, Curigliano G. Investigational platelet-derived growth factor receptor kinase inhibitors in breast cancer therapy. Expert Opin Investig Drugs 2014;23:599610.

(8) Iorio MV, Croce CM. MicroRNA dysregulation in cancer: diagnostics, monitoring and therapeutics. A comprehensive review. EMBO Mol Med 2012;4:143-59.

(9) Zhang J, Chintalgattu V, Shih T, Ai D, Xia Y, Khakoo AY. MicroRNA-9 is an activation-induced regulator of PDGFR-beta expression in cardiomyocytes. J Mol Cell Cardiol 2011;51:337-46.

(10) Ma L, Young J, Prabhala H, Pan E, Mestdagh P, Muth D, et al. miR-9, a MYC/MYCN-activated microRNA, regulates E-cadherin and cancer metastasis. Nat Cell Biol 2010;12:247-56. 
(11) Gwak JM, Kim HJ, Kim EJ, Chung YR, Yun S, Seo AN, et al. MicroRNA-9 is associated with epithelialmesenchymal transition, breast cancer stem cell phenotype, and tumor progression in breast cancer. Breast Cancer Res Treat 2014;147:39-49.

(12) Hill L, Browne G, Tulchinsky E. ZEB/miR-200 feedback loop: at the crossroads of signal transduction in cancer. Int J Cancer 2013;132:745-54.

(13) Meng F, Speyer CL, Zhang B, Zhao Y, Chen W, Gorski DH, et al. PDGFRalpha and beta play critical roles in mediating Foxq1-driven breast cancer stemness and chemoresistance. Cancer Res 2015;75:584-93.

(14) Kong D, Li Y, Wang Z, Banerjee S, Ahmad A, Kim HR, et al. miR-200 regulates PDGF-D-mediated epithelial-mesenchymal transition, adhesion, and invasion of prostate cancer cells. Stem Cells 2009;27:1712-21.

(15) Huang X, Dugo M, Callari M, Sandri M, De CL, Valeri B, et al. Molecular portrait of breast cancer in China reveals comprehensive transcriptomic likeness to Caucasian breast cancer and low prevalence of luminal A subtype. Cancer Med 2015;4:1016-30.

(16) Smyth GK. Linear models and empirical bayes methods for assessing differential expression in microarray experiments. Stat Appl Genet Mol Biol 2004;3:Article3.

(17) Vlachos IS, Hatzigeorgiou AG. Online resources for miRNA analysis. Clin Biochem 2013;46:879-900.

(18) Gualeni AV, Volpi CC, Carbone A, Gloghini A. A novel semi-automated in situ hybridisation protocol for microRNA detection in paraffin embedded tissue sections. J Clin Pathol 2015;68:661-4.

(19) Perou CM, Sorlie T, Eisen MB, van de Rijn M, Jeffrey SS, Rees CA, et al. Molecular portraits of human breast tumours. Nature 2000;406:747-52.

(20) Tang F, Zhang R, He Y, Zou M, Guo L, Xi T. MicroRNA-125b induces metastasis by targeting STARD13 in MCF-7 and MDA-MB-231 breast cancer cells. PLoS ONE 2012;7:e35435.

(21) Burk U, Schubert J, Wellner U, Schmalhofer O, Vincan E, Spaderna S, et al. A reciprocal repression between ZEB1 and members of the miR-200 family promotes EMT and invasion in cancer cells. EMBO Rep 2008;9:582-9. 
(22) Thomson S, Petti F, Sujka-Kwok I, Mercado P, Bean J, Monaghan M, et al. A systems view of epithelial-mesenchymal transition signaling states. Clin Exp Metastasis 2011;28:137-55.

(23) Gravgaard KH, Lyng MB, Laenkholm AV, Sokilde R, Nielsen BS, Litman T, et al. The miRNA-200 family and miRNA-9 exhibit differential expression in primary versus corresponding metastatic tissue in breast cancer. Breast Cancer Res Treat 2012;134:207-17.

(24) Hsu PY, Deatherage DE, Rodriguez BA, Liyanarachchi S, Weng YI, Zuo T, et al. Xenoestrogen-induced epigenetic repression of microRNA-9-3 in breast epithelial cells. Cancer Res 2009;69:5936-45.

(25) Jechlinger $\mathrm{M}$, Sommer A, Moriggl R, Seither $\mathrm{P}$, Kraut $\mathrm{N}$, Capodiecci $\mathrm{P}$, et al. Autocrine PDGFR signaling promotes mammary cancer metastasis. J Clin Invest 2006;116:1561-70.

(26) Kuzmanov A, Hopfer U, Marti P, Meyer-Schaller N, Yilmaz M, Christofori G. LIM-homeobox gene 2 promotes tumor growth and metastasis by inducing autocrine and paracrine PDGF-B signaling. Mol Oncol 2014;8:401-16.

(27) Jurmeister S, Baumann M, Balwierz A, Keklikoglou I, Ward A, Uhlmann S, et al. MicroRNA-200c represses migration and invasion of breast cancer cells by targeting actin-regulatory proteins FHOD1 and PPM1F. Mol Cell Biol 2012;32:633-51.

(28) Lim YY, Wright JA, Attema JL, Gregory PA, Bert AG, Smith E, et al. Epigenetic modulation of the miR200 family is associated with transition to a breast cancer stem-cell-like state. J Cell Sci 2013;126:2256-66.

(29) Kopp F, Oak PS, Wagner E, Roidl A. miR-200c sensitizes breast cancer cells to doxorubicin treatment by decreasing TrkB and Bmi1 expression. PLoS ONE 2012;7:e50469.

(30) Lin J, Liu C, Gao F, Mitchel RE, Zhao L, Yang Y, et al. miR-200c enhances radiosensitivity of human breast cancer cells. J Cell Biochem 2013;114:606-15.

(31) Bai WD, Ye XM, Zhang MY, Zhu HY, Xi WJ, Huang X, et al. MiR-200c suppresses TGF-beta signaling and counteracts trastuzumab resistance and metastasis by targeting ZNF217 and ZEB1 in breast cancer. Int J Cancer 2014;135:1356-68. 
(32) Madhavan D, Zucknick M, Wallwiener M, Cuk K, Modugno C, Scharpff M, et al. Circulating miRNAs as surrogate markers for circulating tumor cells and prognostic markers in metastatic breast cancer. Clin Cancer Res 2012;18:5972-82.

(33) Wang J, Zhao H, Tang D, Wu J, Yao G, Zhang Q. Overexpressions of microRNA-9 and microRNA-200c in human breast cancers are associated with lymph node metastasis. Cancer Biother Radiopharm 2013;28:283-8.

(34) Le MT, Hamar P, Guo C, Basar E, Perdigao-Henriques R, Balaj L, et al. miR-200-containing extracellular vesicles promote breast cancer cell metastasis. J Clin Invest 2014;124:5109-28.

(35) Knezevic J, Pfefferle AD, Petrovic I, Greene SB, Perou CM, Rosen JM. Expression of miR-200c in claudin-low breast cancer alters stem cell functionality, enhances chemosensitivity and reduces metastatic potential. Oncogene 2015;34:5997-6006.

(36) Castilla MA, Diaz-Martin J, Sarrio D, Romero-Perez L, Lopez-Garcia MA, Vieites B, et al. MicroRNA200 family modulation in distinct breast cancer phenotypes. PLOS ONE 2012;7:e47709.

(37) Riaz M, van Jaarsveld MT, Hollestelle A, Prager-van der Smissen WJ, Heine AA, Boersma AW, et al. miRNA expression profiling of 51 human breast cancer cell lines reveals subtype and driver mutation-specific miRNAs. Breast Cancer Res 2013;15:R33.

(38) Ahmad A, Wang Z, Kong D, Ali R, Ali S, Banerjee S, et al. Platelet-derived growth factor-D contributes to aggressiveness of breast cancer cells by up-regulating Notch and NF-kappaB signaling pathways. Breast Cancer Res Treat 2011;126:15-25.

(39) Chen HF, Huang $\mathrm{CH}$, Liu CJ, Hung JJ, Hsu CC, Teng SC, et al. Twist1 induces endothelial differentiation of tumour cells through the Jagged1-KLF4 axis. Nat Commun 2014;5:4697.

(40) Vasudev NS, Reynolds AR. Anti-angiogenic therapy for cancer: current progress, unresolved questions and future directions. Angiogenesis 2014;17:471-94.

(41) Pecot CV, Rupaimoole R, Yang D, Akbani R, Ivan C, Lu C, et al. Tumour angiogenesis regulation by the miR-200 family. Nat Commun 2013;4:2427. 
(42) Zhuang G, Wu X, Jiang Z, Kasman I, Yao J, Guan Y, et al. Tumour-secreted miR-9 promotes endothelial cell migration and angiogenesis by activating the JAK-STAT pathway. EMBO J 2012;31:3513-23. 
Table 1. Relation between PDGFR $\beta$ expression and clinicopathologic features in TNBC human tissues.

\begin{tabular}{|c|c|c|c|c|c|c|}
\hline \multirow{2}{*}{$\begin{array}{l}\text { Clinicopathologic } \\
\text { features }\end{array}$} & \multicolumn{3}{|c|}{ PDGFR $\beta+$ vascular lacunae } & \multicolumn{3}{|c|}{ PDGFR $\beta$ + tumor nest } \\
\hline & $\begin{array}{l}\text { Absence } \\
(\%)\end{array}$ & $\begin{array}{l}\text { Presence } \\
(\%)\end{array}$ & $p$ value & $\begin{array}{l}\text { Absence } \\
(\%)\end{array}$ & $\begin{array}{l}\text { Presence } \\
(\%)\end{array}$ & $p$ value \\
\hline \multicolumn{7}{|l|}{ Histological grade } \\
\hline Grade II & $\begin{array}{l}10 / 45 \\
(22.2)\end{array}$ & $\begin{array}{l}0 / 26 \\
(0)\end{array}$ & 0.0095 & $\begin{array}{l}10 / 42 \\
(23.8)\end{array}$ & $\begin{array}{l}0 / 29 \\
(0)\end{array}$ & 0.0046 \\
\hline \multicolumn{7}{|l|}{ Tumor size } \\
\hline$\geq \mathrm{T} 2$ & $\begin{array}{l}14 / 46 \\
(30.4)\end{array}$ & $\begin{array}{l}11 / 26 \\
(42.3)\end{array}$ & 0.3094 & $\begin{array}{l}15 / 43 \\
(34.9)\end{array}$ & $\begin{array}{l}10 / 29 \\
(34.5)\end{array}$ & 0.9720 \\
\hline \multicolumn{7}{|l|}{ Nodal status } \\
\hline Positive & $\begin{array}{l}16 / 39 \\
(41)\end{array}$ & $\begin{array}{l}12 / 24 \\
(50)\end{array}$ & 0.4863 & $\begin{array}{l}15 / 36 \\
(41.7)\end{array}$ & $\begin{array}{l}13 / 27 \\
(48.1)\end{array}$ & 0.6084 \\
\hline \multicolumn{7}{|l|}{ CD31+ vascular } \\
\hline Presence & $\begin{array}{l}23 / 44 \\
(52.7)\end{array}$ & $\begin{array}{l}25 / 26 \\
(96.1)\end{array}$ & 0.001 & $\begin{array}{l}25 / 41 \\
(61)\end{array}$ & $\begin{array}{l}23 / 29 \\
(79.3)\end{array}$ & 0.1036 \\
\hline \multicolumn{7}{|c|}{ PDGFR $\beta+$ tumor nest } \\
\hline Presence & $\begin{array}{l}6 / 46 \\
(13)\end{array}$ & $\begin{array}{l}23 / 26 \\
(88.5)\end{array}$ & $<0.001$ & & & \\
\hline
\end{tabular}

$p$ values were calculated by chi-square test 
Figure legends.

Figure 1. MiR-9 mediates PDGFRß-induced tube formation ability through STARD13 targeting. (A) TNBC cells were treated with $50 \mathrm{ng} / \mathrm{ml}$ PDGF-BB for $6 \mathrm{~h}$. MiR-9 expression was assessed by qRT-PCR. The result is representative of three independent experiments and expressed as mean \pm standard deviation of three technical replicates. (B) TNBC cells were transfected with either miR-9 precursor or LNA-miR-9, and corresponding controls, for $48 \mathrm{~h}$; tube formation ability was determined as the percentage of modulation in loop formation of treated cells compared to control. (C) MDA-MB-157 cells were transfected with either LNA-miR-9 or LNA-control and, after $24 \mathrm{~h}$, treated or not with PDGF-BB for the following $24 \mathrm{~h}$; tube formation ability was evaluated. (D) MiR-9 expression in basal versus luminal breast cancer was evaluated by qRT-PCR; statistically significant differences were calculated with Mann-Withney test. (E) TNBC cells were transfected with either miR-9 precursor or control for $48 \mathrm{~h}$. STARD13 protein was evaluated by western blot. (F) TNBC cells were transfected with a siRNA specific against STARD13 or a scrambled oligonucleotide for $48 \mathrm{~h}$; tube formation assay was performed (upper panel). Efficiency of silencing was validated by western blot (lower panel). (G) HEK293 cells were co-transfected with either control or miR-9 precursor and with pmiR-Glo dual-luciferase reporter plasmid carrying the wildtype (WT) or the mutated forms (Mut1 and Mut2) of STARD13 3'UTR. Firefly luciferase activity was measured $24 \mathrm{~h}$ after transfection, normalized on Renilla luciferase and expressed as relative value between cells transfected with miR-9 and the corresponding control. ${ }^{*} p<0.05 ; * * p<0.01$; $* * * p<0.001$, Student's t-test.

Figure 2. MiR-200 family inhibits tube formation ability through the suppression of PDGFR 3 . (AB) TNBC cells were transfected with miR-200b, miR-200c or control for $48 \mathrm{~h}$. (A) Tube formation ability was evaluated. (B) PDGFRß and E-cadherin expression was assessed by western blot. (C-D) MDA-MB-231 cells were transfected with either a siRNA against ZEB1 or a scrambled 
oligonucleotide; after $72 \mathrm{~h}$ tube formation ability was analyzed (C); mRNA and protein levels of PDGFR $\beta$ were evaluated by qRT-PCR and western blot, respectively (D). (E) Correlation between ZEB1 and PDGFRB mRNA levels in the TNBC subset of TGCA public dataset. ${ }^{* *} p<0.01$ and $* * * p<0.001$, Student's t-test.

Figure 3. MiR-9 inhibition and miR-200c restoration decrease vasculogenic properties of TNBC tumors. MDA-MB-231 clones (sponge-miR-9 or miR-200c and corresponding controls) were implanted in the m.f.p. of SCID mice. (A) Efficiency of miR-9 inhibition and miR-200c overexpression was validated by reporter assay and qRT-PCR, respectively. For reporter assay, Firefly luciferase was normalized on Renilla luciferase and the luminescence activity expressed as relative value between sponge miR-9 and sponge miR-control. (B) Tumor growth was monitored by caliper measurement. (C) H\&E and CD31 staining of FFPE tumor sections. White arrows indicate tumor cells engaged in vascular lacunae. (D) Quantification of vascular lacunae was performed by counting the total number of CD31-positive vascular structures identified in 5 non overlapping high-power microscopic fields (x400). ${ }^{*} p<0.05 * * p<0.01$ and ${ }^{* * *} p<0.001$, Student's t-test

Figure 4. In vivo treatment with miR-9 inhibitor or miR-200c mimic decreases vasculogenic properties on TNBC. MDA-MB-231 cells were implanted in the m.f.p. of SCID mice. (A) Tumor growth was monitored; black arrows indicate the schedule of treatment. (B) H\&E and CD31 staining of FFPE tumor sections. White arrows indicate tumor cells engaged in vascular lacunae. (C) Quantification of vascular lacunae was performed as described above. (D) ZEB1 staining of FFPE tumor sections. ${ }^{*} p<0.05 ; * * p<0.01$, Student's t-test.

Figure 5. PDGFR $\beta$ identifies vascular lacunae in TNBC tissues. (A) IHC staining reveals tumor cells with reactivity for either CD31 or PDGFR $\beta$ which participate in the formation of vascular structures (white arrows). (B) IF analysis of p53 (red), CD31 (blue) and PDGFRß (green). (C) H\&E and ISH of miR-200c. The ISH signal appeared as brown dots usually localized in the cytoplasm. 
Figure 6. Schematic representation of the network involving PDGFR $\beta$, miR-9, miR-200 and EMT. 